\title{
Key factors affecting ammonium production by an Azotobacter vinelandii strain deregulated for biological nitrogen fixation
}

\author{
Mary H. Plunkett ${ }^{2}$, Carolann M. Knutson ${ }^{2}$ and Brett M. Barney ${ }^{1,2^{*}}$ (D)
}

\begin{abstract}
Background: The obligate aerobe Azotobacter vinelandii is a model organism for the study of biological nitrogen fixation (BNF). This bacterium regulates the process of BNF through the two component NifL and NifA system, where NifA acts as an activator, while NifL acts as an anti-activator based on various metabolic signals within the cell. Disruption of the nifL component in the nifLA operon in a precise manner results in a deregulated phenotype that produces levels of ammonium that far surpass the requirements within the cell, and results in the release of up to $30 \mathrm{mM}$ of ammonium into the growth medium. While many studies have probed the factors affecting growth of $A$. vinelandii, the features important to maximizing this high-ammonium-releasing phenotype have not been fully investigated.

Results: In this work, we report the effect of temperature, medium composition, and oxygen requirements on sustaining and maximizing elevated levels of ammonium production from a nitrogenase deregulated strain. We further investigated several pathways, including ammonium uptake through the transporter AmtB, which could limit yields through energy loss or futile recycling steps. Following optimization, we compared sugar consumption and ammonium production, to attain correlations and energy requirements to drive this process in vivo. Ammonium yields indicate that between 5 and $8 \%$ of cellular protein is fully active nitrogenase MoFe protein (NifDK) under these conditions.
\end{abstract}

Conclusions: These findings provide important process optimization parameters, and illustrate that further improvements to this phenotype can be accomplished by eliminating futile cycles.

Keywords: Azotobacter vinelandii, nifLA, Ammonium, Nitrogen fixation, Optimization

\section{Background}

Azotobacter vinelandii is a diazotrophic (nitrogen fixing), obligate aerobe studied extensively as a model organism for biological nitrogen fixation (BNF), a complex and energy-intensive process that is stringently regulated by the cell [1-4]. BNF is tightly regulated due to the energetically expensive nature of the reaction which consumes a minimum of 16 mols of ATP and 8 mols of electrons per mol of $\mathrm{N}_{2}$ fixed $[4,5]$. Primary expression

*Correspondence: bbarney@umn.edu

${ }^{1}$ Department of Bioproducts and Biosystems Engineering, University of Minnesota, 1390 Eckles Avenue, St. Paul, MN 55108-6130, USA

Full list of author information is available at the end of the article of the genes encoding nitrogenase in $A$. vinelandii is regulated by the nifLA operon clustered in a region of the genome located a significant distance from the nifHDK genes that encode the catalytic subunits of nitrogenase (NifH and NifDK) [6-8]. Previous evidence suggests that NifA acts as an activator of nitrogenase expression, while NifL acts as an anti-activator, sensing available nitrogen, intracellular redox status, and carbon availability within the cell [9]. Several laboratories have disrupted the nifL gene in $A$. vinelandii, resulting in deregulation of nitrogenase expression and subsequent extracellular ammonium accumulation reported to reach up to $30 \mathrm{mM}$ in the supernatant in the late exponential and stationary phases of growth [6, 10-14]. Though much has been elucidated 
in relation to the nature of deregulation of BNF, there remains aspects of this nitrogen-accumulating phenotype that impede a complete understanding of BNF, and how the cell restructures metabolism during this process.

Our laboratory has reconstructed our own version of the nifL deletion resulting in high-ammonium accumulation in the spent medium; A. vinelandii strain AZBB163 [11]. This deregulated strain is a powerful tool to probe the overall changes that occur when $A$. vinelandii redirects substantial amounts of energy and resources toward the primary goal of BNF resulting from increased expression of nitrogenase. We recently completed a global transcriptomic study during peak ammonium production in this deregulated strain, which confirmed dramatic increases in the transcription of nitrogenase genes [6], and also provided a glimpse of how the cell regulated multiple other supporting pathways during ammonium accumulation. This study was important, because it provides an opportunity to study a state that is likely transient in typical diazotrophic growth $[6,15]$.

Azotobacter vinelandii has been studied for many decades in relation to the features associated with diazotrophic growth of the wild-type strain [1, 16-18]. Under diazotrophic culture conditions, BNF is balanced and tightly regulated, assuring that the cell produces only as much nitrogen as is required to sustain its own growth. Factors that affect the rate of BNF in wild-type cells are generally indirectly measured through growth rate or using non-native substrates such as acetylene, that result in a terminal product that can be easily quantified [19, 20]. Deregulated strains of nitrogen fixing bacteria such as AZBB163 are reprogrammed to express nitrogenase regardless of internal nitrogen requirements, and could be a valuable tool in determining the specific factors that might limit BNF in this phenotype. Based on the potential applications of these deregulated strains as an alternative for producing high levels of fixed nitrogen, and the opportunity to measure production directly by monitoring levels of ammonium accumulation in spent culture medium, we thought it important to investigate and characterize the different factors that limit production of ammonium in AZBB163. These studies include environmental parameters related to the culture conditions alone and in tandem with further genomic modifications. We investigated potential competing pathways that we hypothesized would become exacerbated under the conditions associated with the high ammonium accumulating phenotype, and might result in energetically costly and futile cycles that inadvertently limit BNF, such as the ammonium importer, AmtB [21, 22]. A description of these parameters and pathways that were targeted, and the findings associated with them, are presented herein, and demonstrate a potential to further improve ammonium production by the strain, and yield additional products that could further enhance the economic potential of pursuing routes to biofertilizer production through BNF [23-25].

\section{Results \\ The effect of varied temperature on ammonium production}

During initial experiments described here that test specific parameters or genetic manipulations, care was taken to only alter one parameter at a time, and test against the base $A$. vinelandii AZBB163 strain (hereafter referred to as simply AZBB163), which yields high quantities of ammonium. This was done so that differences between individual experiments could be compared to one another and to previous reports $[6,10-12]$. In later experiments, multiple growth parameters and genetic modifications were combined to test specific hypotheses. The AZBB163 strain was constructed to replicate the reports by Bali et al. and Brewin et al. [10, 12]. The first parameter tested was temperature. Ammonium levels achieved by AZBB163 cultures grown at different temperatures varied significantly, with $28{ }^{\circ} \mathrm{C}$ resulting in the highest ammonium concentrations per culture, and $26^{\circ} \mathrm{C}$ resulting in the highest ammonium per culture density (Fig. 1). $\mathrm{OD}_{600}$ values obtained during the culture were not significantly different between the samples grown at $24^{\circ} \mathrm{C}$ and $26^{\circ} \mathrm{C}$, but $28^{\circ} \mathrm{C}$ and $30^{\circ} \mathrm{C}$ resulted in cultures that achieved a slightly elevated $\mathrm{OD}_{600}$. Interestingly, while total ammonium levels increased steadily from 24 to $28{ }^{\circ} \mathrm{C}, 30{ }^{\circ} \mathrm{C}$ resulted in a steep decrease in the rate of ammonium accumulation, indicating that slight increases could have profound effects on the culture and a negative effect above a certain threshold for ammonium yields.

\section{The effect of culture volume on ammonium production}

Azotobacter vinelandii is an obligate aerobe, and given the high ATP demand related to excess nitrogen fixation in AZBB163, we were curious whether oxygen availability may limit growth or hinder nitrogen fixation. A simple approach to increase oxygen availability in batch culture is to decrease the total culture volume within the flask while keeping the flask size constant, providing a greater proportion of exposed surface area to atmosphere versus the volume of culture and more vigorous mixing. Ammonium production was tested in cultures with volumes of $15 \mathrm{~mL}, 30 \mathrm{~mL}$, and $60 \mathrm{~mL}$ each in $125 \mathrm{~mL}$ Erlenmeyer flasks (Fig. 2). All three volumes yielded similar concentrations of ammonium up to $16 \mathrm{~h}$. However, between 16 and $32 \mathrm{~h}$, the cultures significantly deviated with respect to ammonium concentration, with the cultures containing $15 \mathrm{~mL}$ of medium producing the highest concentrations of ammonium, 


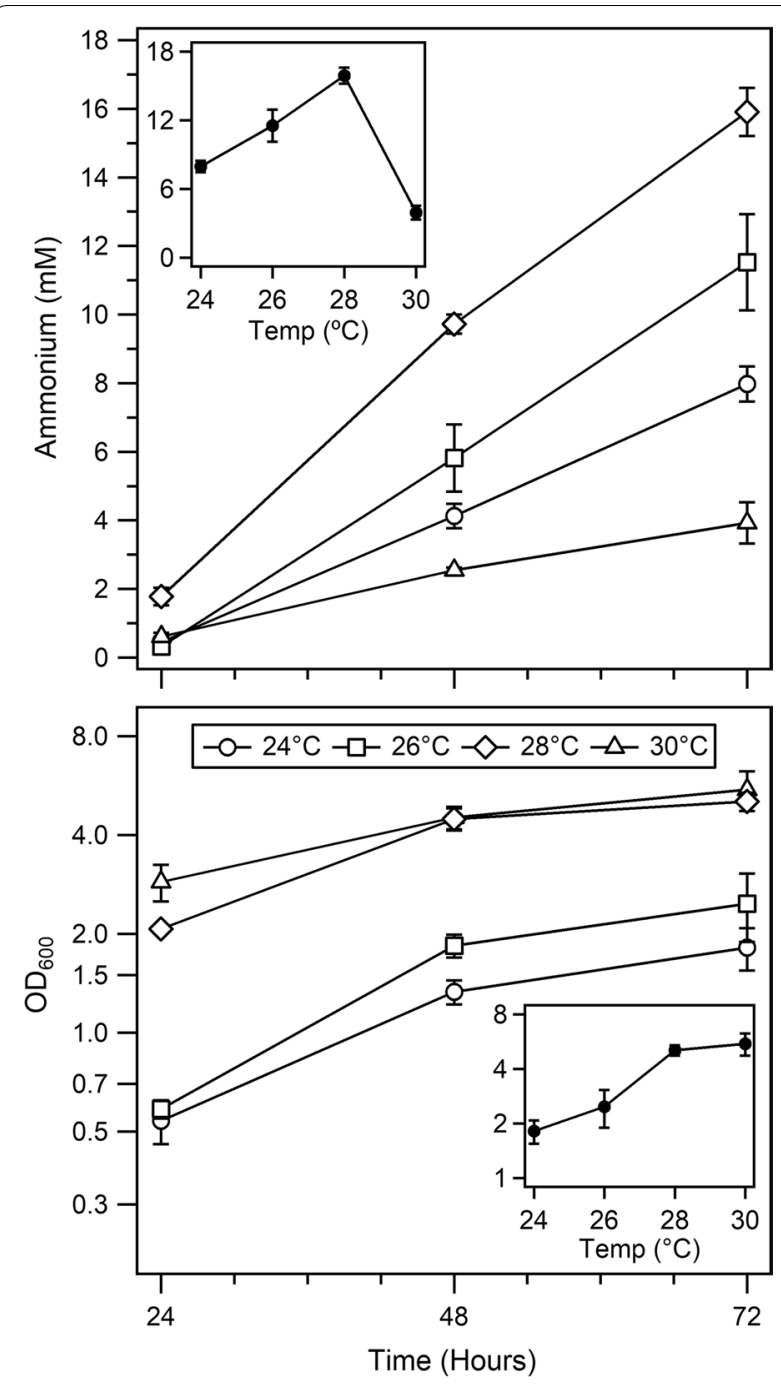

Fig. 1 Ammonium production in AZBB163 grown at varied temperatures. Shown are the ammonium production (top) and $\mathrm{OD}_{600}$ (bottom) for AZBB163 grown at $24^{\circ} \mathrm{C}, 26^{\circ} \mathrm{C}, 28^{\circ} \mathrm{C}$, and $30^{\circ} \mathrm{C}$. Cultures were grown in Burk's medium and were shaken at $180 \mathrm{rpm}$ in $125 \mathrm{~mL}$ Erlenmeyer flasks with $60 \mathrm{~mL}$ of medium. Bottom graph optical density plotted on a $\log _{2}$-based scale (y-axis). Data points indicate averages, while error bars represent standard deviation $(\mathrm{N}=4)$. Insets show the yield obtained at $72 \mathrm{~h}$ (top) and the $\mathrm{OD}_{600}$ obtained at $72 \mathrm{~h}$ (bottom) versus temperature

followed by the $30 \mathrm{~mL}$ cultures, and finally the $60 \mathrm{~mL}$ cultures. All conditions resulted in similar culture densities up to $8 \mathrm{~h}$ of growth, after which the $15 \mathrm{~mL}$ cultures grew more densely, while the $30 \mathrm{~mL}$ and $60 \mathrm{~mL}$ cultures maintained a lower $\mathrm{OD}_{600}$. While variation associated with evaporation rates within cultures of smaller volumes was a concern, analysis revealed that

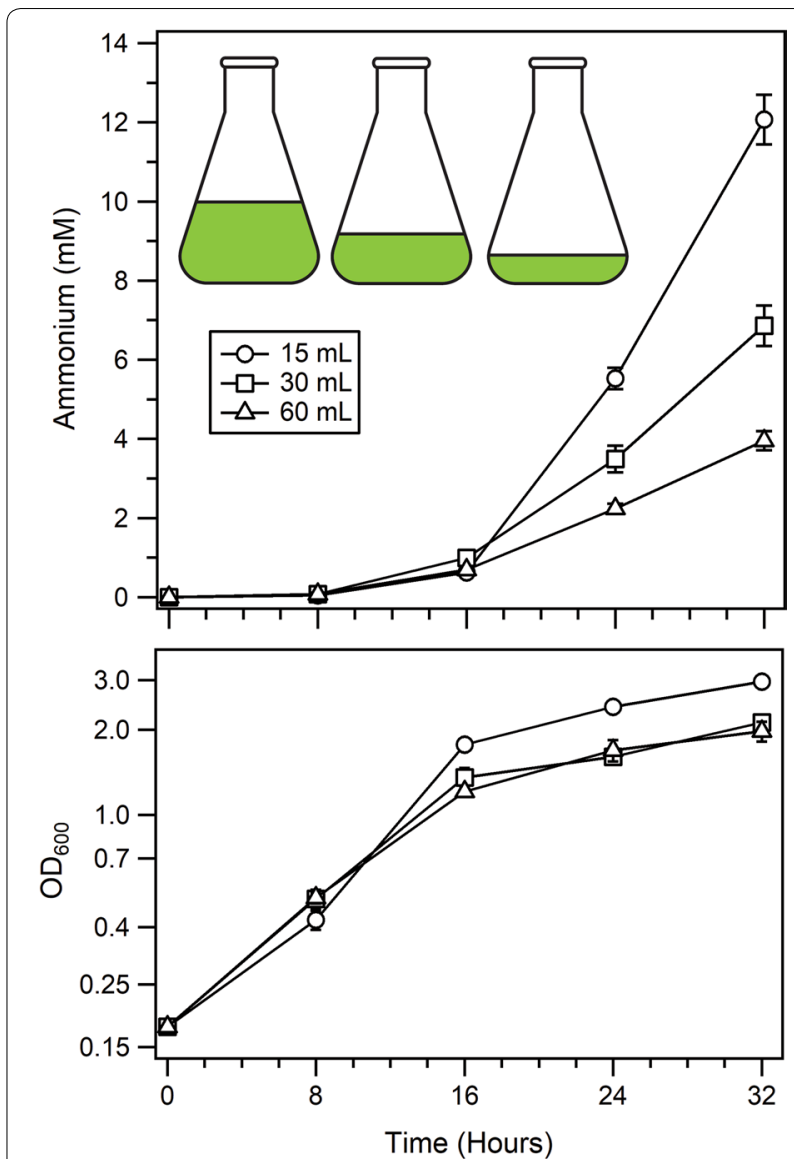

Fig. 2 Ammonium production in AZBB163 in cultures with varied volumes. Shown are the ammonium production (top) and $\mathrm{OD}_{600}$ (bottom) results from growing AZBB163 as $15 \mathrm{~mL}, 30 \mathrm{~mL}$ and $60 \mathrm{~mL}$ cultures. Cultures were grown in Burk's (B) medium with $20 \mathrm{~g} / \mathrm{L}$ starting sucrose concentration at $26^{\circ} \mathrm{C}$ and shaken at $180 \mathrm{rpm}$ in $125 \mathrm{~mL}$ Erlenmeyer flasks. Bottom graph optical density plotted on a $\log _{2}$-based scale (y-axis). Data points indicate averages, while error bars represent standard deviation $(\mathrm{N}=5)$

there was a negligible decrease in culture volume over the duration of the experiment due to evaporation, indicating that the differences in ammonium levels were independent of losses in culture volume based on the experimental design.

Alternative experiments that employed baffled flasks in an effort to further increase the aeration resulted in a sharp drop in levels of ammonium achieved, and increased foaming and cellular aggregation, while efforts to increase aeration further (described below), were not detrimental to cell dispersion and ammonium accumulation. These results indicate that alternative approaches to increase oxygen availability should be approached with caution. 


\section{Disruption of a futile cycle associated with ammonium import}

The ammonium transporter AmtB is constitutively expressed in $A$. vinelandii, regardless of extracellular ammonium levels [6]. In a strain such as AZBB163 that has been deregulated for nitrogen fixation, the active transport of ammonium back into the cell would represent a potentially futile and energy wasting cycle (Fig. 3). We hypothesized that if the $a m t B$ gene were deleted, it might result in a higher rate of ammonium accumulation within the supernatant. Using a strain from a previous report $[11,26]$, the nifL deletion that results in extracellular ammonium accumulation was added to a strain containing the $a m t B$ deletion, creating $A$. vinelandii strain AZBB281. When evaluated for ammonium production, our results demonstrated that AZBB281 accumulated ammonium in the supernatant at a faster rate as compared to AZBB163 at both day 2 and day 3 (Fig. 3), illustrating that this process may represent an energetically wasteful process.
The effect of increased metals and sulfur concentrations on ammonium production

In an effort to ameliorate the potential limitations of essential elements required for cofactor assembly related to nitrogenase, a supplemented medium was developed via a series of experiments testing increases in molybdenum, iron and sulfur $(\mathrm{B} / \mathrm{MoFeS})$; elements essential to metal cluster assembly for nitrogenase component proteins [4]. These preliminary experiments established levels that increased availability of these elements without inducing a detrimental response due to potential toxicity. The adapted medium $(\mathrm{B} / \mathrm{MoFeS})$ balanced an increase in ammonium production with a minimal increase in the concentration of each of these three elements, resulting in the final optimized medium containing sulfur increased threefold, molybdenum fivefold, and iron twofold as compared to standard B medium. AZBB163 was cultured in this medium optimized for high levels of nitrogenase expression (Fig. 4). The results demonstrate a statistically significant $\sim 25 \%$ increase in the amount of
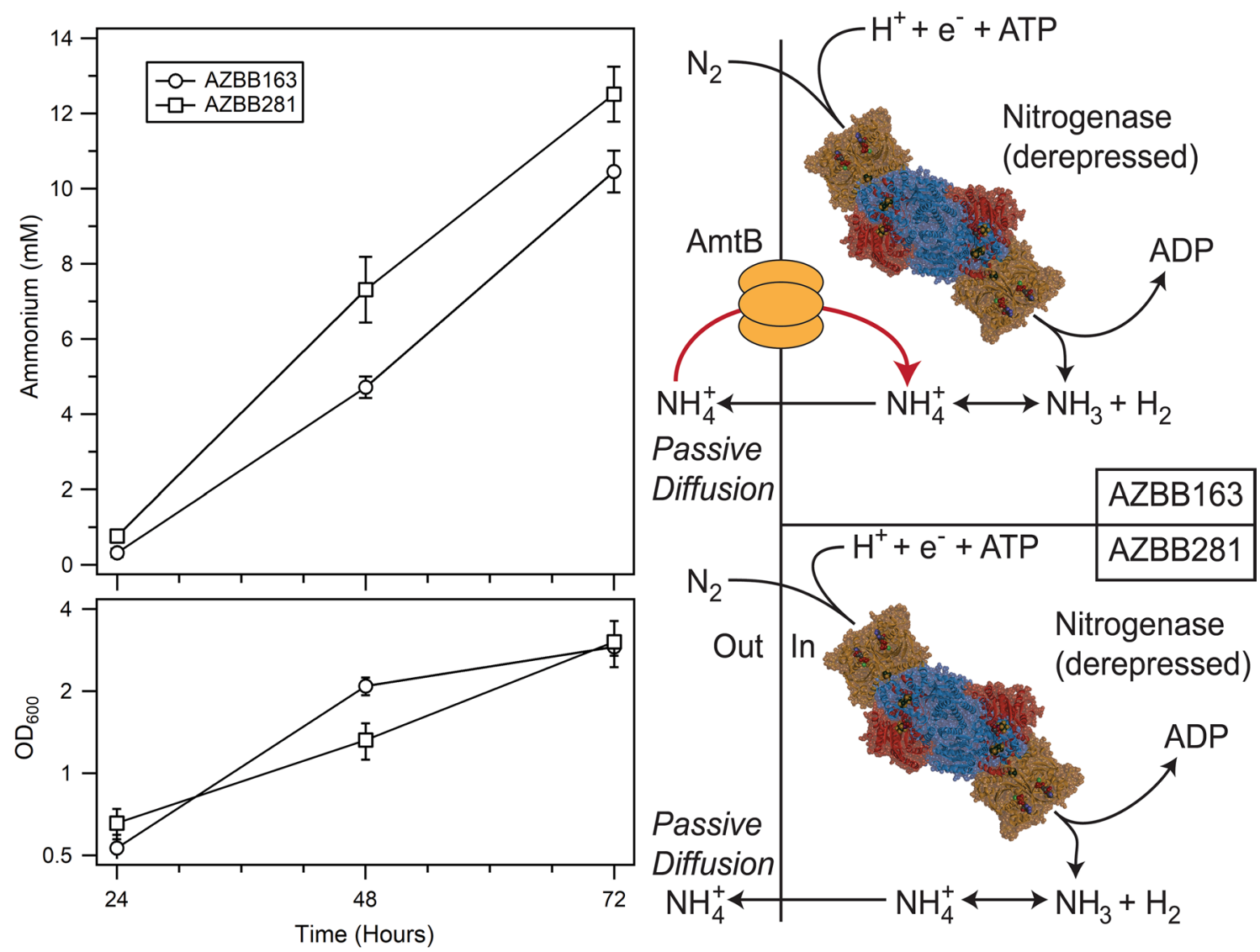

Fig. 3 Ammonium production in a strain lacking the ammonium transporter AmtB. Shown are the ammonium (top left) and OD 600 (bottom left) results of growing AZBB163 and AZBB281 (AZBB163 derivative containing the amtB deletion) in Burk's (B) medium. Shown on the right is a graphical representation of the effects of deleting $a m t B$ within a strain of Azotobacter deregulated for nitrogen fixation. Cultures were grown at $26{ }^{\circ} \mathrm{C}$ and shaken at $180 \mathrm{rpm}$. Bottom graph optical density plotted on a $\log _{2}$-based scale (y-axis). Data points indicate averages, while the error bars represent standard deviation. $\mathrm{N}=4$ 


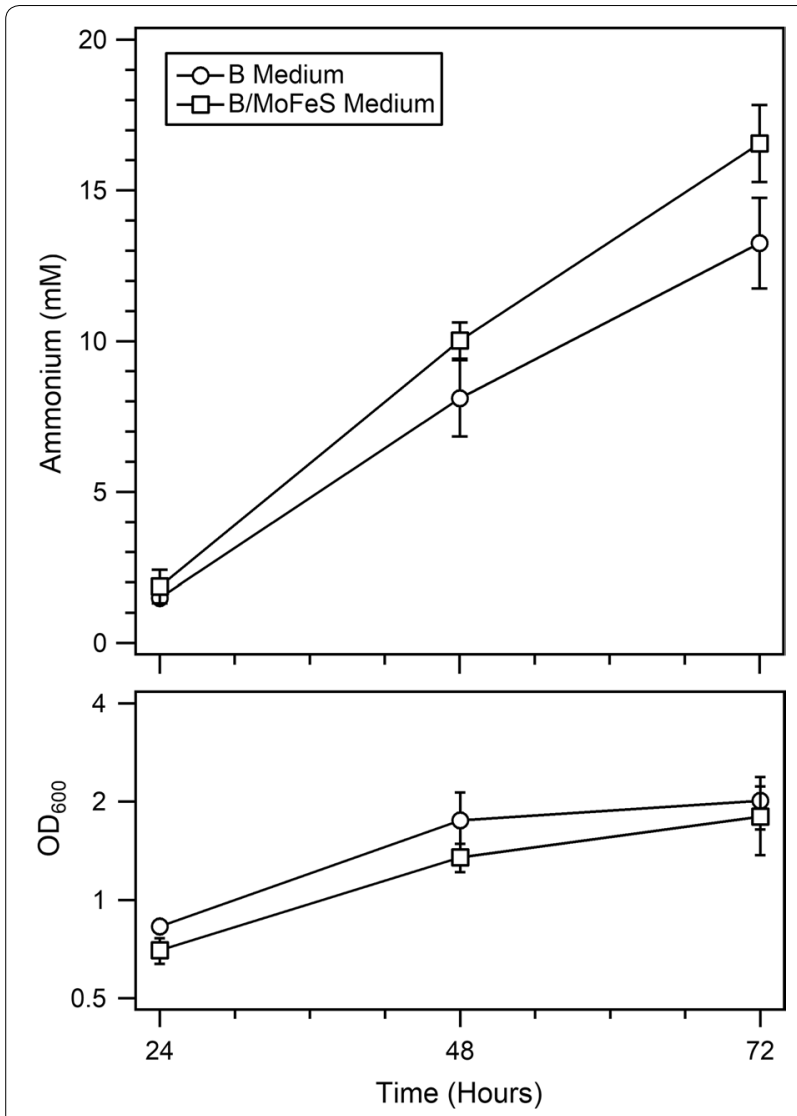

Fig. 4 Ammonium production in AZBB163 grown on Burk's medium versus B/MoFeS medium. Shown are the ammonium production (top) and $\mathrm{OD}_{600}$ (bottom) results from growing AZBB163 in both standard Burk's (B) medium and B/MoFeS medium (supplemented with nitrogenase cofactor-related elements containing twofold increase in iron (Fe), fivefold increase in molybdenum (Mo), and threefold increase in sulfur ( $S$ ) versus B medium). Cultures were grown at $26^{\circ} \mathrm{C}$ and shaken at $180 \mathrm{rpm}$, and were grown as $60 \mathrm{~mL}$ cultures in $125 \mathrm{~mL}$ Erlenmeyer flasks. Bottom graph optical density plotted on a $\log _{2}$-based scale (y-axis). Data points indicate averages, while error bars represent standard deviation $(\mathrm{N}=5)$

ammonium produced on days 2 and 3 of growth, while there was a significant decrease in the $\mathrm{OD}_{600}$ on days 1 and 2 in the presence of optimized cofactor elements $(\mathrm{p} \leq 0.05)$.

\section{Sucrose consumption and limitation in AZBB163}

Our next aim in these experiments was to quantify yields of ammonium in relation to growth substrate utilization. These initial experiments utilized the improved medium containing elevated cofactor elements described above, but remained focused on strain AZBB163. As a first step in this understanding, we looked at rates of ammonium accumulation and substrate consumption over a period of 3 days. Varied
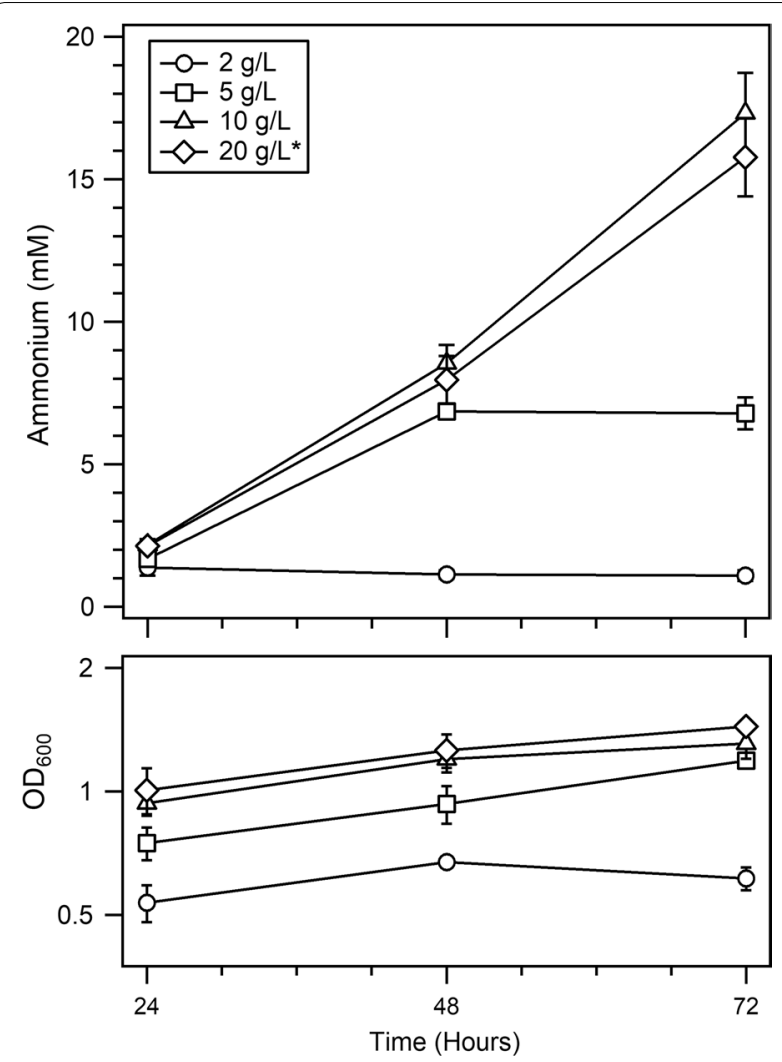

Fig. 5 Ammonium production in AZBB163 grown in B/MoFeS medium with varied concentrations of sucrose. Shown are the ammonium production (top) and $\mathrm{OD}_{600}$ (bottom) results from growing AZBB163 in B/MoFeS medium (Burk's medium supplemented with increased cofactor-related elements) with varied sucrose concentrations, with the standard concentration of sucrose $(20 \mathrm{~g} / \mathrm{L})$ indicated with an $\left(^{*}\right)$. Cultures were grown at $26^{\circ} \mathrm{C}$ and shaken at $180 \mathrm{rpm}$, and were grown as $60 \mathrm{~mL}$ cultures in $125 \mathrm{~mL}$ Erlenmeyer flasks. Bottom graph optical density plotted on a $\log _{2}$-based scale (y-axis). Data points indicate averages, while error bars represent standard deviation $(\mathrm{N}=4)$

concentrations of sucrose in $\mathrm{B} / \mathrm{MoFeS}$ medium were tested in growths of AZBB163, and ammonium levels were analyzed to determine when cultures limited in sucrose would plateau in ammonium production (Fig. 5). B/MoFeS medium was utilized to ensure that sucrose, and not a shortage in the elements related to cofactor assembly, was the limiting factor for growth and ammonium production. The selection of this medium was partially based on limitations in maintaining soluble metals when sugar levels are lower than what is found in standard B medium. Cultures containing $2 \mathrm{~g} / \mathrm{L}$ became limited for sucrose after 1 day of growth, whereas cultures grown in $5 \mathrm{~g} / \mathrm{L}$ of sucrose plateaued in ammonium production after 2 days of growth. The cultures containing $10 \mathrm{~g} / \mathrm{L}$ of sucrose and $20 \mathrm{~g} / \mathrm{L}$ sucrose (the typical concentration in $\mathrm{B}$ 
medium) generated very similar levels of extracellular ammonium and culture density on all 3 days of the experiment.

\section{Ammonium yields versus sucrose consumption under optimized conditions}

One question arising from the results described above related to the trade off in ammonium produced versus sucrose consumed. Results provided in Fig. 5 revealed that ammonium levels approaching $20 \mathrm{mM}$ were achieved with half of the sucrose that is typically required to reach $25-30 \mathrm{mM}$ ammonium (see below). In order to more accurately profile the rate of sucrose consumption under optimized conditions, initial sucrose concentrations in $\mathrm{B} / \mathrm{MoFeS}$ medium were lowered to $10 \mathrm{~g} / \mathrm{L}$ and cultures were grown beyond the point of sucrose depletion using strain AZBB281, the strain lacking the futile cycle for ammonium recycle (Fig. 6). After 3 days, nearly all sucrose was consumed from the medium, and increases in ammonium concentration had plateaued.

The highest rates of ammonium production and sucrose consumption occurred between 22 and $57 \mathrm{~h}$. The ratio of mmols of ammonium produced per mmol of sucrose consumed was $0.93 \pm 0.07$ during this window

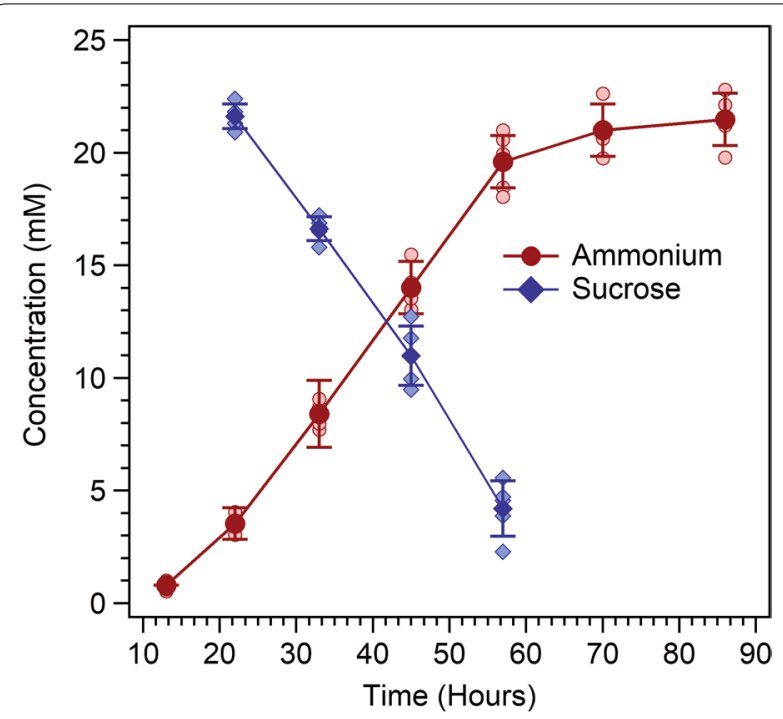

Fig. 6 Ammonium production and sucrose consumption in AZBB281 grown in sugar-limited ( $29 \mathrm{mM}$ Sucrose) B/MoFeS medium. Shown are the ammonium production and sucrose consumption results obtained when growing AZBB281 in Burk's medium supplemented with cofactor-related elements (B/MoFeS medium) with $10 \mathrm{~g} / \mathrm{L}$ of starting sucrose concentration (1/2 of sucrose concentration in typical B medium). Cultures were grown at $26^{\circ} \mathrm{C}$ and shaken at $200 \mathrm{rpm}$ in $125 \mathrm{~mL}$ Erlenmeyer flasks with $60 \mathrm{~mL}$ of medium. Data points indicate averages, while error bars represent standard deviation $(\mathrm{N}=5)$. All data points (lighter shade) are included for reference of time. Under these conditions, the cultures were inoculated into medium containing approximately $29 \mathrm{mM}$ of sucrose and ceased accumulation of ammonium at concentrations of $21.5 \pm 1.1 \mathrm{mM}$ ammonium, making the net conversion approximately $1 \mathrm{~mol}$ ammonium produced per 1.35 mol sucrose consumed.

\section{Maximizing rates of ammonium production}

As a final experiment, we sought to incorporate each of the identified optimal conditions with the optimized genetic construct to compare the improvements that could be achieved (Fig. 7). Our goal in this experiment differed from those described above. In this experiment, we sought to determine the highest rate of ammonium accumulation in the extracellular space, regardless of the

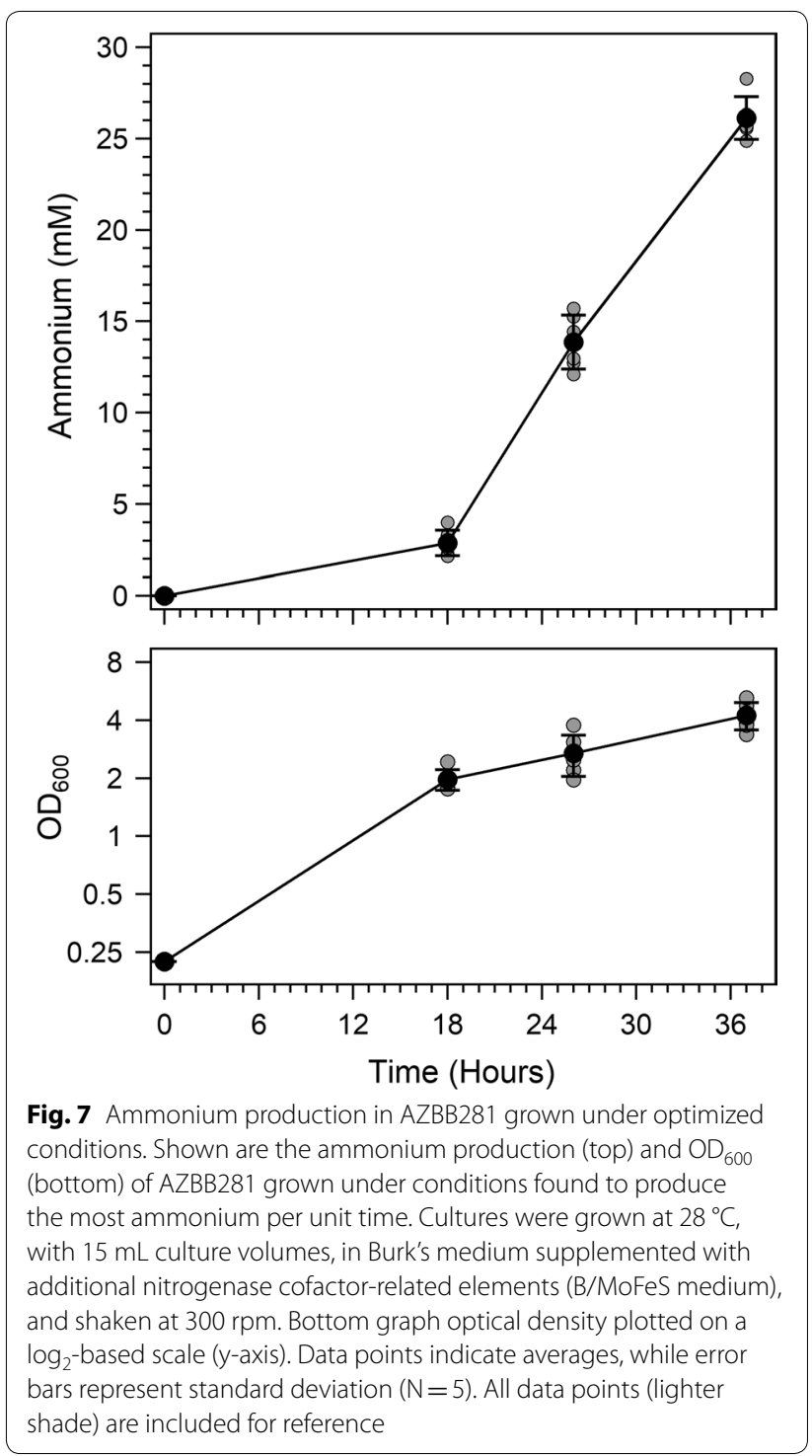


sucrose required to achieve these rates. Strain AZBB281 was grown at $28{ }^{\circ} \mathrm{C}$, in $15 \mathrm{~mL}$ of $\mathrm{B} / \mathrm{MoFeS}$ medium. These combined conditions yielded the highest rate of ammonium production over time from any of these experiments. The experiment was run at $300 \mathrm{rpm}$ until it reached an ammonium level of greater than $25 \mathrm{mM}$. During the 8 -h period between 18 and $26 \mathrm{~h}$ for the experiment, cultures achieved rates of approximately $1.37 \mathrm{mM}$ of extracellular ammonium accumulated per hour. This value is equivalent to nearly $23 \mu \mathrm{M}$ per minute $(23 \mathrm{nmol} /$ min per $\mathrm{mL}$ ). Protein levels between 18 and $26 \mathrm{~h}$ averaged about $300 \mu \mathrm{g} / \mathrm{mL}$, indicating an ammonium yield of about $75 \mathrm{nmol} / \mathrm{min} /(\mathrm{mg}$ total cellular protein). To yield $23 \mathrm{nmol} / \mathrm{min}$ of ammonium with purified nitrogenase enzyme requires approximately $38 \mu \mathrm{g}$ of MoFe protein (NifDK) with an excess of Fe protein (NifH) [27, 28]. Based on these calculations, $8-13 \%$ of the cellular protein is required as MoFe protein (NifDK) functioning at optimal rates with full activity [27, 29]. Achieving these rates in vitro required 10 molar equivalents of Fe protein to MoFe protein [28], while others have reported Fe:MoFe protein ratios of $\sim 2: 1$ within $A$. vinelandii wild-type cells during maximum in vivo nitrogenase activity [30]. These protein levels agree with previous findings related to protein expression profiles for these BNF deregulated strains [6].

\section{Discussion}

Optimizing growth parameters to maximize the production of desired products is a simple, yet robust approach that could compliment what more difficult genetic manipulations are able to achieve. In this work, both culture conditions and further genetic manipulations were employed independently and in combination to yield further increases in the accumulation of ammonium in A. vinelandii strains partially deregulated for BNF. These studies provide insight into the limiting factors that A. vinelandii may face when deregulated for BNF and producing copious quantities of extracellular ammonium. The growth parameters investigated in this study included oxygen limitation, temperature, and availability of the Mo, Fe and S for the biosynthesis of cofactors essential for nitrogenase. One aspect that became evident as a result of these findings is that there is a balancing act that must be taken into consideration when seeking to optimize conditions to maximize either yields or rate of ammonium production through BNF in $A$. vinelandii.

We approached the questions regarding the role of individual growth parameters through an incremental strategy, altering only one parameter in each experiment, and retaining the common growth parameters for all other aspects of growth. Modifying the growth temperature had a profound and intriguing effect on the amount of ammonium produced. The highest temperature tested $\left(30^{\circ} \mathrm{C}\right)$ was found to be detrimental to ammonium accumulation in the extracellular space versus slight decreases in culture temperature, and resulted in a trade-off in limiting ammonium while increasing culture density (Fig. 1 ). Growing cultures at a slightly lower temperature of $28^{\circ} \mathrm{C}$ resulted in the highest rates of ammonium accumulation in the medium. Lowering the temperature to $26^{\circ} \mathrm{C}$ resulted in similarly high ammonium levels, but lowered culture density, and growth at $24{ }^{\circ} \mathrm{C}$ gave similarly lowered culture density, but lower levels of ammonium, while still achieving higher levels of ammonium than cultures grown at $30{ }^{\circ} \mathrm{C}$. Typical growth temperatures for culturing $A$. vinelandii are often cited as $30^{\circ} \mathrm{C}$ in the primary literature [31-34], and $30{ }^{\circ} \mathrm{C}$ is the suggested growth parameters described by the American Type Cultures Collection (ATCC).

This suggested temperature of $30{ }^{\circ} \mathrm{C}$ for culturing $A$. vinelandii differs from optimal conditions for respiration described by Lineweaver, Burk and Horner more than 80 years ago, who found that $A$. vinelandii respires at increasingly higher rates up to $35^{\circ} \mathrm{C}$, after which point respiration rates decrease [1]. The effect of temperature on nitrogen fixation in various bacteria has been studied previously by probing a wide range of temperatures using the alternative substrate acetylene, and found optimal rates of activity at $35^{\circ} \mathrm{C}$ for Azotobacter chroococcum [35]. Additional studies have looked at the regulation of nitrogenase related gene expression at different temperatures in A. vinelandii [36, 37]. In general, the activity of isolated nitrogenase enzymes tends to increase with steadily higher temperatures through $45^{\circ} \mathrm{C}[38,39]$. Most species of Azotobacter produce only as much ammonium as is necessary to sustain their own growth. Since many assays of nitrogenase activity done in vivo actually use the substrate acetylene, which has characteristics that differentiate it from $\mathrm{N}_{2}$, the strain used in this study provides a unique means of testing the effect of temperature on BNF in a strain specifically deregulated for this process. This optimal temperature for ammonium production contrasts with prior reports. This is not completely unexpected, as the metabolism within this strain has been altered dramatically to achieve this ammonium production phenotype [6, 10-12, 29].

The shift in cell density that occurred between 26 and $28{ }^{\circ} \mathrm{C}$ was also unexpected (Fig. 1). We previously reported differences in final cell densities that were obtained between the AZBB163 and the wild-type strain [6]. The stark change that occurred in AZBB163 during this rather narrow temperature range could result from several different sources, including a shift of metabolism that favors polyhydroxybutyrate production or a limitation of other key intracellular metabolites, which both 
fell outside the scope of this current study. The selection of an optimal temperature with AZBB163 and similar strains derived from AZBB163 is thus dependent on the goals of the experiment. If optimal rates of ammonium accumulation were the goal, then $28{ }^{\circ} \mathrm{C}$ would be ideal. However, in the majority of our experiments, we aimed for high ammonium yields from a minimal mass of cells, and in those experiments, we thus selected $26^{\circ} \mathrm{C}$.

Increased cell density should translate to increased competition for available oxygen. It is well established that $A$. vinelandii expends a great deal of energy attempting to carefully modulate internal oxygen levels $[1,40]$. Since oxygen solubility in water is actually quite low versus many other gases, and $A$. vinelandii is known to have one of the highest respiratory rates reported for bacteria $[1,18]$, it is quite possible that oxygen availability could limit BNF and the excess ammonium production that results in the ammonium-releasing phenotype that is associated with AZBB163. In contrast, nitrogenase is an oxygen-sensitive enzyme, and elevated levels of oxygen near the active site of nitrogenase could irreversibly damage this enzyme. This conflicting nature of these two highly dependent processes are at odds with one another. Thus, the AZBB163 strain offered a unique opportunity to determine how well the cell protects these elevated levels of nitrogenase under elevated expression conditions and whether the strain was in fact oxygen limited as a result.

Outside of using a fermenter or other forced aeration device, there are several manners to improve oxygen delivery to batch-grown flask cultures. One approach would be to increase the fraction of oxygen within the atmosphere. However, since $A$. vinelandii also needs the nitrogen from the atmosphere in these experiments to support BNF, such an approach was viewed as self-sabotaging. We thus selected only natural atmospheric compositions of oxygen and nitrogen gases. The addition of baffles to the Erlenmeyer flasks used in these studies was also viewed as a favorable method of introducing more oxygen, but resulted in significant foaming and aggregation of the cells, accompanied by a dramatic drop in ammonium production. Reduction of culture volume, while keeping the flask size constant, is a classical way to provide more oxygen to batch grown cultures by increasing the surface area exposed to atmosphere, resulting in increased aeration [41]. The approach of lowering the volume of culture while maintaining the same size of Erlenmeyer flask clearly demonstrated that ammonium production could be steadily and significantly increased by lowering the ratio of volume versus the available surface area exposed to the standard oxygen atmosphere (Fig. 2). This illustrated that the culture was still oxygen limited under our initial conditions, including those we have reported previously $[6,11]$, and that the strain is well adapted to protect the nitrogenase from any detrimental effects associated with the oxygen, even with the elevated expression of nitrogenase that accompanies this phenotype [6,12].

In a previous study investigating the changes in global transcription that occur within AZBB163 [6], we determined that molybdenum availability limited ammonium accumulation in AZBB163. The production of the full complement of metal clusters in each full Mo-based nitrogenase complex $(\mathrm{NifDK}+2 \mathrm{NifH})$ requires $38 \mathrm{Fe}$ and $2 \mathrm{Mo}$, but also requires $40 \mathrm{~S}$ atoms. Each cluster is bound to the enzyme through one or more cysteine ligands. This would also increase the demand for S. Based on the elevated levels of expression of both NifDK and $\mathrm{NifH}$ that were found from our prior analysis [6], and the relatively low concentration of sulfate included in the general recipe for Burk's medium [42], we hypothesized that sulfur might also limit ammonium production in AZBB163. Experiments revealed that elevating the levels of both Mo, Fe and S, independently and in combination, resulted in a slight improvement in ammonium accumulation. Based on these observations, a new medium was formulated that increased levels of Mo by $5 \times$, Fe by $2 \times$ and $S$ by $3 \times$. The optimized medium resulted in an increase in ammonium accumulation as compared to standard Burk's medium (Fig. 4). Though standard Burk's medium provides sufficient levels of the microelements required to maintain the diazotrophic phenotype for the wild-type strain, the elevated cofactor requirements of AZBB163 to sustain high levels of extracellular ammonium production necessitates an increase in sulfur. Our prior studies with AZBB163 indicated a limitation due to metal depletion by the cell, which manifested as an increase in transcription for siderophore peptides [6, 43], but diminished upon addition of excess metals. These results thus indicate that all three micronutrients involved in the biosynthesis of nitrogenase cofactors should be considered when optimizing BNF through AZBB163 and related strains.

Prior efforts revealed that elimination of the gene $a m t B$, which codes for an ammonium transporter, results in the release of ammonium to the extracellular culture medium that is sufficient to support the co-culture of algae [11]. AmtB is thought to serve a role in A. vinelandii to shuttle ammonium into the cell by linking this transport to the activity of glutamine synthetase, resulting in the hydrolysis of ATP, which also serves a vital role in BNF [4]. It has also been shown that ammonium and methylamine transport in $A$. vinelandii is dependent on membrane potential $[44,45]$. For this reason, any strains manipulated to produce elevated levels of extracellular ammonium are potentially wasting ATP or energy 
associated with maintaining the membrane potential in an effort to recycle that ammonium back into the cell in a process that should be futile and wasteful. Additionally, since this process is not directly coupled to BNF, it could result in a much higher waste of energy than what would be expected for deletion of the hydrogenase, especially as levels of ammonium accumulate outside of the cell. Contrary to what was found when deleting the hydrogenases $[29,46]$, the deletion of $a m t B$ did result in an improvement in the rate of ammonium accumulation (Fig. 3), indicating that this process is likely wasting unnecessary energy and limiting ammonium accumulation.

Our final experiments pursued here combined the collective understanding of the physical parameters and genetic modifications to determine both maximum yields and maximal rates of ammonium generated through AZBB281. This strain contains both the nifL disruption that results in deregulation of nitrogen fixation along with the $a m t B$ deletion that disrupts any futile attempts to transport extracellular ammonium back into the cell. Strains were grown under two sets of conditions. The first approach sought to maximize yield of ammonium per mol of sucrose (Fig. 6). In the second approach (Fig. 7), the goal was to determine maximum rates of yielded per unit time. Our experiment was performed at $300 \mathrm{rpm}$ and $28{ }^{\circ} \mathrm{C}$ in the improved $\mathrm{B} / \mathrm{MoFeS}$ medium in $125 \mathrm{~mL}$ Erlenmeyer flasks containing $15 \mathrm{~mL}$ of media, which resulted in rates of extracellular ammonium accumulation per hour that surpassed any of the results found in any of the prior experiments (Figs. 1, 2, $3,4,5$ and 6). In these experiments, rates of extracellular ammonium accumulation surpassed $1 \mathrm{mM}$ per hour, and increased at a relatively linear rate between hours 18 and 37. In these experiments, we provided the standard quantities of sucrose $(\sim 58 \mathrm{mM})$ to the medium, and achieved ammonium levels averaging $26 \mathrm{mM}$ at $37 \mathrm{~h}$. This increased rate of ammonium accumulation came at a cost of higher sucrose consumption, as nearly all of the sucrose was consumed by hour 37 ( 90\%). This indicates an ammonium yield of about $0.5 \mathrm{mM}$ ammonium per mole of sucrose, which is lower than what we found with lower quantities of sucrose when aeration and rate of ammonium accumulation was not maximized (Fig. 6). These results highlight the trade-off that is associated with higher rates versus required energy in the form of the sugar substrate, and the maximum concentrations of ammonium that the strain could obtain in liquid culture.

In most of our efforts to test maximal yields of ammonium, the strains are generally able to achieve levels approaching $25-30 \mathrm{mM}$ ammonium, when provided sufficient levels of sucrose. This $30 \mathrm{mM}$ maximum range appears to represent a proverbial "brick wall," as ammonium levels greater than this value were difficult to obtain, even when sufficient sucrose levels remained in the culture. Thus, slowing the rate of oxygen delivery, and as a result, ammonium accumulation, required $\sim 1.4 \mathrm{~mol}$ of sucrose per mol of ammonium (Fig. 6), while increasing oxygen delivery to decrease the amount of time required to accumulate slightly elevated levels of ammonium required $\sim 2.3 \mathrm{mols}$ of sucrose per mol of ammonium (Fig. 7).

\section{Conclusions}

This report demonstrates potential levels of ammonium yielded under our current best-case scenario for ammonium accumulation by an aerobic organism. It further demonstrates that transport processes recycling ammonium, such as those linked to AmtB, may actually hinder ammonium production rates as a result of futile cycles for a deregulated strain. Yields of ammonium per mole of sucrose illustrate that tradeoffs exist between maximizing rates of ammonium production versus optimizing total yields per mole of sucrose. As a result of these new constructs, an alternative full cell assay is now possible that eliminates the need to study nitrogenase activity through a surrogate such as acetylene, and as a result, we demonstrate that temperatures long believed to be optimal for growth of $A$. vinelandii are actually detrimental to maximizing ammonium production in a BNF deregulated strain.

\section{Methods}

Genetic constructs, gene deletion and confirmation Azotobacter vinelandii DJ (ATCC BAA-1303) was obtained from Dennis Dean (Virginia Tech) and cultured on Burk's medium [42]. Escherichia coli JM109 was acquired from New England Biolabs (Ipswich, MA) and utilized to construct the plasmids described. Plasmids used to construct final vectors for gene deletions in $A$. vinelandii are described in Table 1 . Primers used for confirmation of genetic constructs are described in Table 2. Detailed methods for the transformation of A. vinelandii have been described previously $[26,42]$. Transformation of $A$. vinelandii DJ in order to modify target genes utilizing double homologous recombination with the $p y r F$ counter-selection technique were implemented as described previously [11, 26]. Details for strains constructed for use in this study are provided in Table 3. Methods describing the construction of strains AZBB106, and AZBB163 have been described previously $[11,29]$. 
Table 1 Key plasmid constructs utilized in this work

\begin{tabular}{|c|c|c|c|}
\hline Plasmid $^{a}$ & Relevant gene(s) cloned or plasmids manipulated & Parent vector & $\begin{array}{l}\text { Source } \\
\text { or references }\end{array}$ \\
\hline pPCRNH3-44 & $\begin{array}{l}\text { Incorporated } \mathrm{C} \text { to } \mathrm{T} \text { mutation into kanamycin cassette region to create } \mathrm{Nif}^{+} \\
\text {phenotypes with high ammonium production }\end{array}$ & pPCRNH3-43 & {$[6]$} \\
\hline PPCRPYRF1 & Cloned pyrF and flanking regions from $A$. vinelandii & pBB053 & {$[26]$} \\
\hline
\end{tabular}

a The sequences of all plasmids in this study are available upon request

Table 2 Key primers used in this study

\begin{tabular}{lll}
\hline Primer & Sequence $\mathbf{5}^{\prime} \mathbf{- 3}^{\prime}$ & Purpose \\
\hline BBP950 & GAGCACACCCATCACGGTCAGAG & nifLA modification confirmation \\
BBP1322 & GATCTCCATCGACTCGATCTTGTCCAGGGTGAAC & nifLA modification confirmation \\
BBP2006 & CACGTGCCAGGAATTCCTCCATG & amtB gene deletion confirmation \\
BBP2007 & CTGTGGACGATGGCCAGGGACATGGATC & amtB gene deletion confirmation \\
\hline
\end{tabular}

Table 3 Key strains constructed and utilized in this study

\begin{tabular}{|c|c|c|c|}
\hline A. vinelandii strain & Genetic features & Plasmid utilized & Parent strain \\
\hline DJ (ATCC BAA-1303) & $\begin{array}{l}\text { Wild-type A. vinelandii with diminished alginate production resulting in ease of transforma- } \\
\text { tion }\end{array}$ & None & None \\
\hline AZBB109 & $\triangle a m t B$ & pPCRPYRF ${ }^{a}$ & AZBB106 ${ }^{\mathrm{a}}$ \\
\hline$A Z B B 163^{b *}$ & $\begin{array}{l}\text { nifL:: } \operatorname{Kan}^{\mathrm{R}}(\mathrm{pPCRNH3}-43) \text {. Was grown in medium without nitrogen source, and mutated to a } \\
\text { nif+ phenotype that also produces high levels of extracellular ammonia }\end{array}$ & $\begin{array}{l}\text { pPCRNH3-43, } \\
\text { spontaneous } \\
\text { mutation }\end{array}$ & AZBB150 \\
\hline AZBB281* & $\triangle a m t B$, nifl::Kan ${ }^{R}(p P C R N H 3-44)$ & pPCRNH3-44c & AZBB109 \\
\hline
\end{tabular}

$\mathrm{Kan}^{\mathrm{R}}$ Kanamycin resistance

Strains indicated by an asterisk $\left(^{*}\right)$ are completed and were used in ammonium production experiments

a As described previously [26]

b As described previously [11]

c As described previously [6]

\section{Growth of cells and B/MoFeS medium development}

Azotobacter vinelandii strains were grown in base/acid washed $125 \mathrm{~mL}$ Erlenmeyer flasks containing $60 \mathrm{~mL}$ of medium unless otherwise stated. Experiments measuring the effect of increased aeration were performed by inoculating a larger volume of sterile medium, and then adding aliquots of different volumes of medium into sterile flasks of the same size $(125 \mathrm{~mL})$. All experiments were performed with at least three replicates. Burk's $/ \mathrm{Na}_{2} \underline{\mathrm{MoO}_{4}}$, $\underline{\mathrm{FeSO}_{4}}, \mathrm{Na}_{2} \underline{\mathrm{SO}}_{4}(\mathrm{~B} / \mathrm{MoFeS})$ medium was developed using standard Burk's (B) medium recipes [42] supplemented with $2 \times$ iron $\left(18 \mu \mathrm{M}\right.$ increased to $36 \mu \mathrm{M}$ as $\left.\mathrm{FeSO}_{4}\right), 5 \times$ molybdenum (1 $\mu \mathrm{M}$ increased to $5 \mu \mathrm{M}$ as $\left.\mathrm{Na}_{2} \mathrm{MoO}_{4}\right)$, and $3 \times$ sulfur $\left(0.8 \mathrm{mM}\right.$ increased to $2.4 \mathrm{mM}$ as $\left.\mathrm{Na}_{2} \mathrm{SO}_{4}\right)$ as determined through a series of experiments testing ranges of these elements. In each experiment, cultures were inoculated with approximately equal initial concentrations of cells $\left(\mathrm{OD}_{600} \sim 0.15-0.25\right)$ within each particular experiment from cells scraped off $B$ plates containing appropriate antibiotics grown for 2 days at $30{ }^{\circ} \mathrm{C}$. Cultures were grown at $26{ }^{\circ} \mathrm{C}$ with agitation at $180 \mathrm{rpm}$ unless otherwise noted. The temperature of $26{ }^{\circ} \mathrm{C}$ was chosen as a standard due to the balance of ammonium produced per culture optical density, which was maximal at this temperature. Supernatant was collected by centrifugation $(>12,000 \mathrm{~g}$ for $1 \mathrm{~min})$ of samples at the indicted time points.

\section{Ammonium quantification}

Assays for ammonium quantification used the colorimetric o-phthalaldehyde method as described previously utilizing a Cary 50 Bio Spectrophotometer measuring absorbance at $412 \mathrm{~nm}[6]$.

\section{Sucrose quantification}

For experiments measuring the levels of sucrose in the medium, the Sigma-Aldrich kit (Part No: MAK013-1KT) was used as directed by the manufacturer. 


\section{Protein quantification}

To quantify the total amount of starting protein, isolated cell pellets were suspended in $1 \mathrm{~mL}$ of water and sonicated for $60 \mathrm{~s}$ (Misonix LX-2000, Qsonica, Newtown, CT) inside of a $2.0 \mathrm{~mL}$ Eppendorf tube, and centrifuged at 13,000 $\mathrm{g}$ for $5 \mathrm{~min}$ to remove cell debris. Cell lysate was then added to $1.0 \mathrm{~mL}$ of Coomassie Plus (Bradford) assay reagent (Pierce, Rockford, IL), mixed by pipetting and incubated at room temperature for $20 \mathrm{~min}$. Absorbance was read at a wavelength of $595 \mathrm{~nm}$ (Varian Inc., Palo Alto, CA). Samples were compared to a standard curve prepared using Bovine Serum Albumin as a standard (Pierce, Rockford, IL).

\section{Abbreviation}

BNF: Biological nitrogen fixation.

\section{Authors' contributions}

All authors conceived and designed experiments, collected and analyzed data and wrote the manuscript. All authors read and approved the final manuscript.

\section{Funding and acknowledgements}

This work was supported by Grants from the MnDRIVE (MnDrive 12) transdisciplinary research initiative through the University of Minnesota based on funding from the state of Minnesota to B.M.B. and the from the National Institute of Food and Agriculture (Project Numbers MIN-12-070 and MIN-12-081), and award number 2020-67019-31148 through the United States Department of Agriculture. M.H.P. and C.M.K. were further supported through funding from the National Science Foundation (CBET-1437758).

\section{Availability of data and materials}

All data generated or analyzed during this study are included in this published article or in prior published reports.

\section{Ethics approval and consent to participate}

Not applicable.

\section{Consent for publication}

Not applicable.

\section{Competing interests}

The authors are inventors on US Patent 9,796,957, Genetically modified diazotrophs and methods of using same.

\section{Author details}

${ }^{1}$ Department of Bioproducts and Biosystems Engineering, University of Minnesota, 1390 Eckles Avenue, St. Paul, MN 55108-6130, USA. ${ }^{2}$ Biotechnology Institute, University of Minnesota, St. Paul, MN 55108, USA.

Received: 20 January 2020 Accepted: 4 May 2020

Published online: 19 May 2020

\section{References}

1. Lineweaver $\mathrm{H}$, Burk D, Horner CK. The temperature characteristic of respiration of Azotobacter. J Gen Physiol. 1932;15(5):497-505.

2. Shah VK, Ugalde RA, Imperial J, Brill WJ. Molybdenum in nitrogenase. Annu Rev Biochem. 1984;53:231-57.

3. Smith BE. Structure. Nitrogenase reveals its inner secrets. Science. 2002;297(5587):1654-5.

4. Barney BM, Lee HI, Dos Santos PC, Hoffman BM, Dean DR, Seefeldt LC. Breaking the $\mathrm{N}_{2}$ triple bond: insights into the nitrogenase mechanism. Dalton Trans. 2006;19:2277-84.
5. Burgess BK, Lowe DJ. Mechanism of molybdenum nitrogenase. Chem Rev. 1996;96(7):2983-3011.

6. Barney BM, Plunkett MH, Natarajan V, Mus F, Knutson CM, Peters JW Transcriptional analysis of an ammonium-excreting strain of Azotobacter vinelandii deregulated for nitrogen fixation. Appl Environ Microbiol. 2017;83(20):e01534-17.

7. Hamilton TL, Ludwig M, Dixon R, Boyd ES, Dos Santos PC, Setubal JC, et al. Transcriptional profiling of nitrogen fixation in Azotobacter vinelandii. J Bacteriol. 2011;193(17):4477-86.

8. Setubal JC, dos Santos P, Goldman BS, Ertesvåg H, Espin G, Rubio LM, et al. Genome sequence of Azotobacter vinelandii, an obligate aerobe specialized to support diverse anaerobic metabolic processes. J Bacteriol. 2009;191(14):4534-45.

9. Little R, Martinez-Argudo I, Dixon R. Role of the central region of NifL in conformational switches that regulate nitrogen fixation. Biochem Soc Trans. 2006:34:162-4.

10. Bali A, Blanco G, Hill S, Kennedy C. Excretion of ammonium by a nifL mutant of Azotobacter vinelandii fixing nitrogen. Appl Environ Microbiol. 1992;58(5):1711-8.

11. Barney BM, Eberhart LJ, Ohlert JM, Knutson CM, Plunkett MH. Gene deletions resulting in increased nitrogen release by Azotobacter vinelandii: application of a novel nitrogen biosensor. Appl Environ Microbiol. 2015;81(13):4316-28

12. Brewin $B$, Woodley $P$, Drummond $M$. The basis of ammonium release in nifl mutants of Azotobacter vinelandii. J Bacteriol. 1999:181(23):7356-62.

13. Ortiz-Marquez JC, Do Nascimento M, Curatti L. Metabolic engineering of ammonium release for nitrogen-fixing multispecies microbial cell-factories. Metab Eng. 2014;23:154-64.

14. Ortiz-Marquez JC, Do Nascimento M, Dublan MD, Curatti L. Association with an ammonium-excreting bacterium allows diazotrophic culture of oil-rich eukaryotic microalgae. Appl Environ Microbiol. 2012;78(7):2345-52

15. Schreiber F, Littmann S, Lavik G, Escrig S, Meibom A, Kuypers MMM, et al. Phenotypic heterogeneity driven by nutrient limitation promotes growth in fluctuating environments. Nat Microbiol. 2016;1(6):7.

16. Burk D, Horner CK, Lineweaver $\mathrm{H}$. Injury and recovery of respiration and catalase activity in Azotobacter. J Cell Comp Physiol. 1932;1(3):435-49.

17. Newton JW, Wilson PW, Burris RH. Direct demonstration of ammonia as an intermediate in nitrogen fixation by Azotobacter. J Biol Chem. 1953:204(1):445-51.

18. Jensen HL. The azotobacteriaceae. Bacteriol Rev. 1954;18(4):195-214.

19. Koch B, Evans HJ, Russell S. Properties of the nitrogenase system in cellfree extracts of bacteroids from soybean root nodules. Proc Natl Acad Sci USA. 1967;58(4):1343-50.

20. Guth $J \mathrm{H}$, Burris $\mathrm{RH}$. Inhibition of nitrogenase-catalyzed $\mathrm{NH}_{3}$ formation by $\mathrm{H}_{2}$. Biochemistry. 1983;22(22):5111-22.

21. Meletzus D, Rudnick P, Doetsch N, Green A, Kennedy C. Characterization of the glnK-amtB Operon of Azotobacter vinelandii. J Bacteriol. 1998:180(12):3260-4

22. Martinez-Argudo I, Little R, Shearer N, Johnson P, Dixon R. The NifL-NifA system: a multidomain transcriptional regulatory complex that integrates environmental signals. J Bacteriol. 2004;186(3):601-10.

23. Ambrosio R, Ortiz-Marquez JCF, Curatti L. Metabolic engineering of a diazotrophic bacterium improves ammonium release and biofertilization of plants and microalgae. Metab Eng. 2017:40:59-68.

24. Apte SK, Chaurasia AK. Improved eco-friendly recombinant Anabaena sp. strain PCC7120 with enhanced nitrogen biofertilizer potential. Appl Environ Microbiol. 2011;77(2):395-9.

25. Leaungvutiviroj $C$, Ruangphisarn $P$, Hansanimitkul P, Shinkawa H, Sasaki K. Development of a new biofertilizer with a high capacity for $\mathrm{N}_{2}$ fixation, phosphate and potassium solubilization and auxin production. Biosci Biotechnol Biochem. 2010;74(5):1098-101.

26. Eberhart LJ, Knutson CM, Barney BM. A methodology for markerless genetic modifications in Azotobacter vinelandii. J Appl Microbiol. 2016;120(6):1595-604.

27. Barney BM, Igarashi RY, Dos Santos PC, Dean DR, Seefeldt LC. Substrate interaction at an iron-sulfur face of the FeMo-cofactor during nitrogenase catalysis. J Biol Chem. 2004;279(51):53621-4.

28. Barney BM, Lukoyanov D, Igarashi RY, Laryukhin M, Yang TC, Dean DR, et al. Trapping an intermediate of dinitrogen $\left(\mathrm{N}_{2}\right)$ reduction on nitrogenase. Biochemistry. 2009:48(38):9094-102 
29. Knutson CM, Plunkett MH, Liming RA, Barney BM. Efforts toward optimization of aerobic biohydrogen reveal details of secondary regulation of biological nitrogen fixation by nitrogenous compounds in Azotobacter vinelandii. Appl Microbiol Biotechnol. 2018;102(23):10315-25.

30. Poza-Carrión C, Jiménez-Vicente E, Navarro-Rodríguez M, EchavarriErasun C, Rubio LM. Kinetics of nif gene expression in a nitrogen-fixing bacterium. J Bacteriol. 2014;196(3):595-603.

31. Hill S, He LH, Kennedy C. Physiological characterisation of an Azotobacter vinelandii nifU-deletion mutant and its spontaneous $\mathrm{Nif}^{+}$revertants that over-produce cytochrome bd. FEMS Microbiol Lett. 1999;175(2):185-91.

32. Martin AE, Burgess BK, lismaa SE, Smartt CT, Jacobson MR, Dean DR. Construction and characterization of an Azotobacter vinelandii strain with mutations in the genes encoding flavodoxin and ferredoxin I. J Bacteriol. 1989;171(6):3162-7.

33. Strandberg GW, Wilson PW. Formation of the nitrogen-fixing enzyme system in Azotobacter vinelandii. Can J Microbiol. 1968;14(1):25-31.

34. Wong TY, Maier RJ. $\mathrm{H}_{2}$-dependent mixotrophic growth of $\mathrm{N}_{2}$-fixing Azotobacter vinelandii. J Bacteriol. 1985;163(2):528-33.

35. Jain DK, Beyer D, Rennie RJ. Dinitrogen fixation $\left(\mathrm{C}_{2} \mathrm{H}_{2}\right.$ reduction) by bacterial strains at various temperatures. Plant Soil. 1987;103(2):233-7.

36. Miller RW, Eady RR. Molybdenum and vanadium nitrogenases of Azotobacter chroococcum-low temperature favours $\mathrm{N}_{2}$ reduction by vanadium nitrogenase. Biochem J. 1988;256(2):429-32.

37. Walmsley J, Kennedy C. Temperature-dependent regulation by molybdenum and vanadium of expression of the structural genes encoding three nitrogenases in Azotobacter vinelandii. Appl Environ Microbiol. 1991;57(2):622-4.

38. Dilworth MJ, Eady RR. Hydrazine is a product of dinitrogen reduction by the vanadium-nitrogenase from Azotobacter chroococcum. Biochem J. 1991;277:465-8.
39. Dilworth MJ, Eldridge ME, Eady RR. The molybdenum and vanadium nitrogenases of Azotobacter chroococcum: effect of elevated temperature on $\mathrm{N}_{2}$ reduction. Biochem J. 1993;289:395-400.

40. Inomura K, Bragg J, Follows MJ. A quantitative analysis of the direct and indirect costs of nitrogen fixation: a model based on Azotobacter vinelandii. ISME J. 2017;11(1):166-75.

41. Finn RK. Agitation-aeration in the laboratory and in industry. Bacteriol Rev. 1954;18(4):254-74.

42. Dos Santos PC. Molecular biology and genetic engineering in nitrogen fixation. Methods Mol Biol. 2011;766:81-92.

43. Wichard T, Bellenger JP, Morel FMM, Kraepiel AML. Role of the siderophore azotobactin in the bacterial acquisition of nitrogenase metal cofactors. Environ Sci Technol. 2009;43(19):7218-24.

44. Barnes EM Jr, Zimniak P. Transport of ammonium and methylammonium ions by Azotobacter vinelandii. J Bacteriol. 1981;146(2):512-6.

45. Ludewig U, Neuhäuser B, Dynowski M. Molecular mechanisms of ammonium transport and accumulation in plants. FEBS Lett. 2007;581(12):2301-8.

46. Barney BM. Aerobic nitrogen-fixing bacteria for hydrogen and ammonium production: current state and perspectives. Appl Microbiol Biotechnol. 2020;104(4):1383-99.

\section{Publisher's Note}

Springer Nature remains neutral with regard to jurisdictional claims in published maps and institutional affiliations.
Ready to submit your research? Choose BMC and benefit from:

- fast, convenient online submission

- thorough peer review by experienced researchers in your field

- rapid publication on acceptance

- support for research data, including large and complex data types

- gold Open Access which fosters wider collaboration and increased citations

- maximum visibility for your research: over 100M website views per year

At BMC, research is always in progress.

Learn more biomedcentral.com/submissions 